\title{
Junction formation during desiccation cracking
}

\author{
K. B. Toga and B. Erdem Alaca* \\ Department of Mechanical Engineering, Koc University, Rumeli Feneri Yolu, 34450 Sariyer, Istanbul, Turkey
}

(Received 18 November 2005; revised manuscript received 4 July 2006; published 9 August 2006)

\begin{abstract}
In order to provide a sound physical basis for the understanding of the formation of desiccation crack networks, an experimental study is presented addressing junction formation. Focusing on junctions, basic features of the network determining the final pattern, provides an elemental approach and imparts conceptual clarity to the rather complicated problem of the evolution of crack patterns. Using coffee-water mixtures a clear distinction between junction formation during nucleation and propagation is achieved. It is shown that for the same drying suspension, one can switch from the well-known symmetric triple junctions that are unique to the nucleation phase to propagation junctions that are purely dictated by the variations of the stress state. In the latter case, one can even manipulate the path of a propagating crack in a deterministic fashion by changing the stress state within the suspension. Clear microscopic evidence is provided for the formation of propagation junctions, and material inhomogeneity is observed to be reflected by a broad distribution of angles, in stark contrast to shrinkage cracks in homogeneous solid films.
\end{abstract}

DOI: 10.1103/PhysRevE.74.021405

PACS number(s): 82.70.Kj, 62.20.Mk, 62.20.Dc

\section{INTRODUCTION}

Pattern formation during desiccation cracking has been extensively observed in laboratory settings using aqueous suspensions of a variety of materials ranging from clay [1-3], coffee [4], and starch [5] to alumina [6], silica [7], or latex [8] particles and different compounds such as nickel phosphate and ferric ferrocyanide [9] spread on substrates such as glass. Similar observations are also made on mud in natural settings [10-13]. Modeling efforts, on the other hand, are directed towards the prediction of the evolution of polygonal crack patterns as a function of material properties and boundary conditions $[14,15]$ with a particular interest in the estimation of crack spacings [16-18].

The dependence of crack patterns on suspension thickness and interfacial properties is a point that is generally agreed upon. First of all, a reduced thickness is observed to lead to wavy cracks. This can be related to the disturbance of the sharp drying front within the suspension as the thickness goes down [19], which is incorporated in numerical models by increasing the disorder in the system [20]. Other studies relate this phenomenon to the fact that nucleation of cracks is facilitated by increased rate of drying as the thickness is reduced. As a consequence, cracks screen each other and propagation is hampered [4]. On the other hand, as the thickness is increased, propagation is preferred over nucleation, since this time stress relaxation becomes pronounced [21].

Reduced interface friction, on the other hand, is observed to lead to increased spacing between cracks $[4,19]$. The tractions that disappear at the crack face build up over a longer distance if slip occurs at the interface, and hence, cracking cannot take place in this region of reduced stresses, leading to increased spacings between cracks. The size of this region is incorporated in various numerical models as a reference length [20,22].

Limiting our scope to the studies that are specifically concerned with junction formation, the following observations are found to be noteworthy.

\footnotetext{
*Electronic address: ealaca@ku.edu.tr FAX: +90-212-338-1548.
}

(i) For suspensions of coffee [4] or polystyrene-blockpoly(acrylic acid) diblock copolymer micelles [19], a transition of the mean value of the joining angle between cracks from around $120^{\circ}$ to around $90^{\circ}$ is observed to take place with increasing thickness of the suspension.

(ii) However, similar experiments on alumina nanoparticles did not yield any evidence of $120^{\circ}$ junctions except for the early stages where sand particles added to the suspension served as crack nucleation sites with three cracks nucleating symmetrically [6].

(iii) This was further supported by experiments on clay soil where an early development of $120^{\circ}$ junctions was observed to come to a halt at later stages [13].

In this study, a clear picture based on experiments on coffee-water mixtures will be presented addressing the origin of such differences concerning junction formation. Approaching crack patterns with an emphasis on their basic elements, junctions, is found to provide a clear methodology for a complicated problem. First of all, compared to the vast number of published reports on pattern formation during desiccation, only a few experimental studies mention the distinction without supplying a satisfactory explanation. One reason for the lack of available data is the fact that nucleation and propagation cannot be studied separately due to experimental limitations; i.e., experiments do not allow a complete isolation of the nucleation phase from propagation. However, it will be shown that by patterning the surface of the substrate with stress raisers, nucleation junctions can be studied in isolation from propagation junctions. Furthermore, propagation can be suppressed completely if the distance between the stress raisers is kept small enough to maximize energy release during nucleation.

The second aspect missing in conventional desiccation experiments besides separation of nucleation and propagation is the lack of control on the stress state within the drying medium. Addressing this issue is crucial for understanding why cracks branch during propagation. For this purpose, a second set of experiments is designed where one has close control of the water concentration and distribution within the drying suspension. This allows complete determination of 
stress distribution, and hence, establishing a relation between stresses and resulting crack paths, if there exists any, is facilitated. Once this is established, one can argue that in the case of an uncontrolled drying experiment, crack paths should still be governed by the same mechanism. Hence, kinks, diversions from straight paths frequently observed under ambient (uncontrolled) conditions, are adjustments of crack paths to inhomogeneous stresses around them.

Finally, after the mechanism of kinking is discussed, the relation between kinking and junction formation during propagation is established, and the paper is concluded with a third set of experiments where junction formation during propagation is observed in situ, showing that kinks, being sites of higher stresses, nucleate the third arm of the junction. To our knowledge, this is the only microscopic evidence of junction formation during propagation in the literature.

\section{COMPARISON OF INITIATION AND PROPAGATION PHASES}

At this point one should note that the works by Shorlin et al. [6] and Vogel et al. [13] are some of the few experimental reports clearly distinguishing junction formation during nucleation from that during propagation. As far as nucleation is concerned, one might argue that points of disturbance on the surface of a drying suspension, such as a bird's footprint or a gastropod trail [23] on drying mud, can act as stress raisers and, hence, these points are preferred sites for crack nucleation [Fig. 1(a)]. Simultaneous nucleation of triple cracks can be further related to the minimization of the potential energy of the cracking medium by generating the least amount of new surfaces through drawing parallels between fracture and other examples of pattern formation in nature such as bubble clusters [24].

Triple junctions during propagation, on the other hand, form either when the sharp elbow that forms as a result of kinking serves as an additional crack initiator or when a propagating crack meets an existing crack with $90^{\circ}$ [Fig. 1(b)]. The amount of kinking that a crack undergoes is dictated merely by the local stress distribution near the crack tip; i.e., the crack changes its direction to maximize its opening stress. By saying so, we should make clear that, on the one hand, the disorder in systems we are considering is not high enough for fragmentation by the coalescence of initial defects to take over the fragmentation by crack growth [20]. On the other hand, there should be enough inhomogeneity causing changes in the stress state that leads a crack to kink from its initial path. This inhomogeneity derives from variations in fracture strength of the suspension, interface friction between suspension and substrate, or solvent concentration. Coffee:water suspensions (1:2 by mass) on poly(methyl methacrylate) (PMMA) substrates used in this study are observed to exhibit the right amount of inhomogeneity so that cracks propagate by channeling rather than by coalescence, and they often kink. The final thickness of dried suspension is measured to be around $3 \mathrm{~mm}$ in every experiment.

In the experiment of Fig. 1(a), a network of needles is used to pattern the surface of the PMMA substrate prior to casting the suspension. Distance between needles is kept

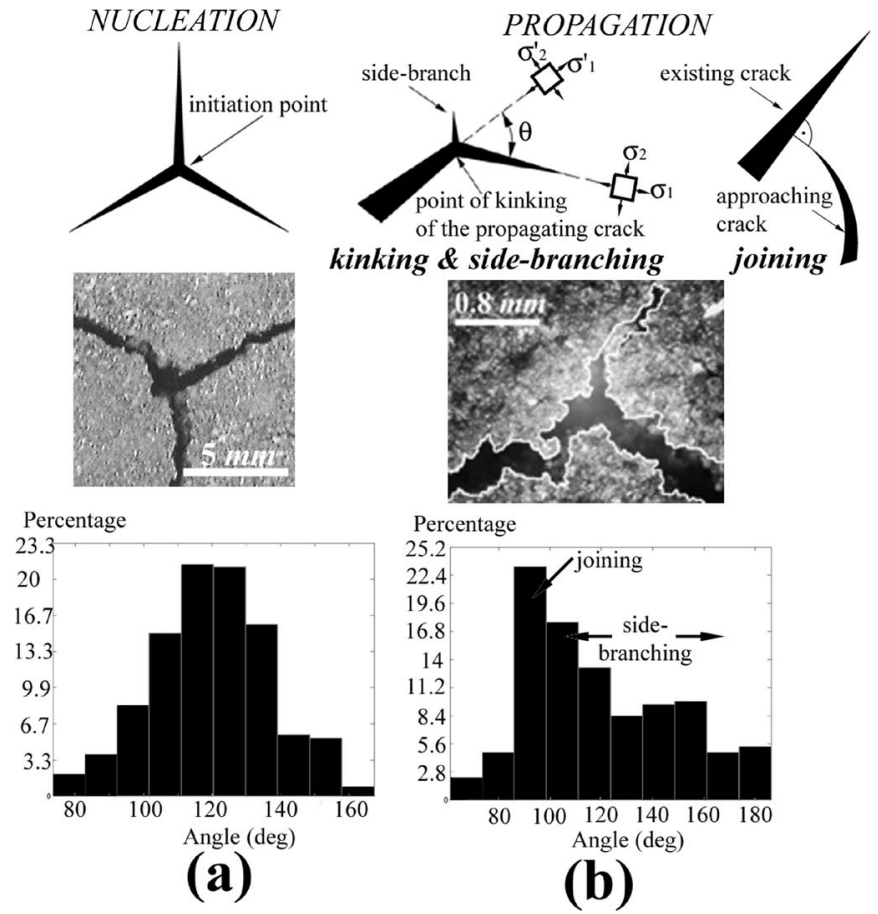

FIG. 1. (a) Nucleation-dominated cracking. Triple junctions with symmetric cracks are observed. The photograph depicts three cracks emanating from a hole created using a needle as a stress raiser in a drying coffee-water mixture. The fact that the pattern in this particular sample is nucleation dependent is reflected by the angle distribution, where the average junction angle is around $120^{\circ}$. (b) Propagation-dominated cracking. Triple junctions in this case are created either when the kink point of a propagating crack serves as a stress raiser and initiates a side branch as shown in the micrograph or when a propagating crack meets an existing crack with $90^{\circ}$. In sidebranching, the kink angle $\theta$, being a function of local stress distribution and fracture toughness, does not necessarily lead to the symmetric picture of (a). If the stress distribution is known, $\theta$ can be calculated by finding the direction of the maximum opening stress, $\sigma_{2}$, at the crack tip. Therefore, the rather uniform distribution of junction angles in this case is an indication of the inhomogeneity within the system-i.e., the existence of variations in fracture strength, interface friction, or solvent concentration.

small enough to suppress crack propagation. Once cracks initiate at one particular point, stresses around its immediate vicinity are relieved, and hence, neighboring cracks cannot propagate into the relaxed region. During drying, each needle serves as such a nucleation point, and this is reflected by the dominance of $120^{\circ}$ in the histogram of junction angles.

On the other hand, if we do not pattern the surface and let the crack network evolve under ambient conditions, kinking and side branching and joining with $90^{\circ}$ are observed as in Fig. 1(b). The peak angle is observed to be $90^{\circ}$, indicating the dominance of joining cracks in forming triple junctions. Other angles are due to kinking and side branching and, to a certain extent, due to nucleation. As expected, the rather broad distribution indicates the inhomogeneity in the system, in particular the stress state, with the kink angle $\theta$ taking a variety of values. In contrast to this, systems of reduced inhomogeneity, usually solid coatings such as glazed surfaces 


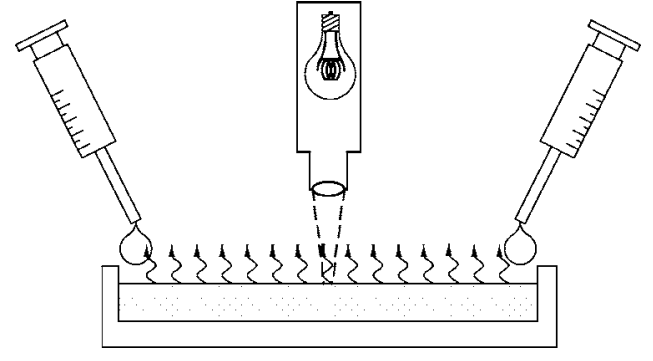

FIG. 2. Experimental setup and boundary conditions. The center of the circular coffee:water mixture container is heated by focusing light at the midpoint. The perimeter is always kept wet so that water diffuses within the suspension from the perimeter towards the center, while it also evaporates; hence, a constant water concentration gradient is achieved between the maximum (perimeter) and minimum (center) concentration spots.

on ceramics, do not exhibit sharp kinks, and almost all junction angles are observed to be $90^{\circ}$, while only a minority of $120^{\circ}$ triplets exist [25-27].

\section{STRESS ANALYSIS AND KINK FORMATION}

Claims in the preceding discussion are to be verified by finding out whether the stress state indeed dictates crack paths in the coffee:water mixture as it does in solid media. For this purpose, a two-dimensional experiment is designed next, where the stress distribution within the suspension does not evolve under ambient conditions, but certain boundary conditions for temperature and water concentration are imposed that lead to a well-controlled, specific stress state. If the resulting crack paths follow maximum stress lines, this will verify that cracking in this particular system is a rather deterministic phenomenon.

Conditions imposed in the experiment are depicted in Fig. 2 and explained below: A circular container of PMMA with radius $R=4.5 \mathrm{~cm}$ is filled with the aforementioned coffeewater mixture, and the center of the sample is heated by focusing light on a region with a diameter of approximately $1 \mathrm{~cm}$. In the beginning of the experiment, the sample is in the form of a uniform colloidal suspension. As evaporation takes place over the surface of the sample, water is introduced into the system at the perimeter so that the perimeter of the circle is always kept wet, while the central region gradually loses its water content. Ultimately, when the steady state is reached, the mixture is still in the form of a colloidal suspension around the edge, whereas it has evolved into an elastic continuum elsewhere. Hence, the mass concentration of water varies along the radial direction, acquiring a minimum at the center. Light merely serves the purpose of accelerating this process. However, it is to be noted that once cracking takes place, cracks serve as new evaporation paths for water and disturb the uniformity of the sample [28]. Hence, the formulation of this section will be valid until the onset of cracking.

The problem will be modeled in the framework of simple Fick's diffusion assuming coupling between thermal gradients and mass transfer to be negligible. The radial

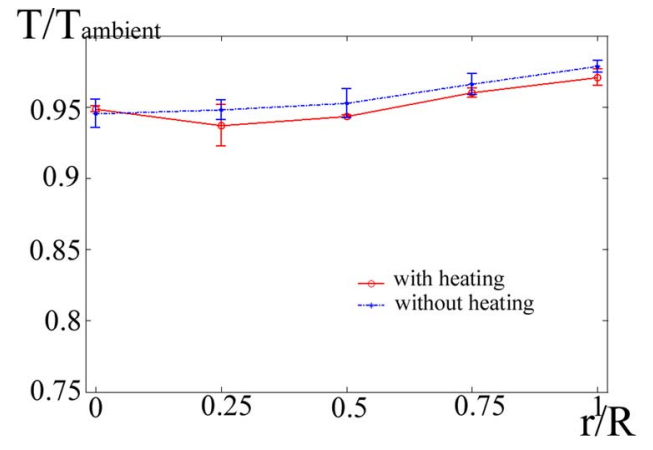

FIG. 3. (Color online) Steady-state temperature distribution measured in ${ }^{\circ} \mathrm{C}$ as a function of radial coordinate $r$. In the sample with no heating, evaporative cooling is apparent in the form of a monotonous decrease of temperature, where the perimeter is kept at a higher temperature due to continuous feeding of water, while the central region cools down due to evaporation. Nevertheless, the maximum temperature difference between the edge and the center in the absence of heating does not exceed $2{ }^{\circ} \mathrm{C}$.

distribution of temperature given in Fig. 3 also justifies its minor effect. Once the distribution of water concentration inside the sample is determined, the resulting stresses will be calculated using elasticity.

The distribution of water concentration within the suspension can be evaluated by considering the in-plane diffusion of water from the perimeter towards the center and evaporation into air over the sample surface. Due to the symmetry of the problem, the mass flux of water per unit time and per unit area, $m_{w}$, within the suspension is a function of the radial coordinate $r$ only. Hence, it can be written as

$$
m_{w}=-D_{w} \frac{d C_{w}}{d r},
$$

where $D_{w}$ is the associated diffusion coefficient and $C_{w}$ is the mass concentration of water per unit volume.

On the other hand, to calculate the rate of evaporation per unit area, it is assumed that water vapor has to diffuse through a stagnant air column. Driven by the concentration gradient through the column, the diffusion is assumed to be independent of convection, and hence, Stefan's law can be utilized to relate total evaporation rate to diffusion and bulk mass flow of water vapor [29]:

$$
m_{e}=-D_{e} \frac{M_{w}}{R_{0} T}\left(\frac{p}{p-p_{w}}\right) \frac{d p_{w}}{d z},
$$

where $m_{e}$ and $D_{e}$ are the mass flux of water and diffusion coefficient for evaporation, respectively. $M_{w}$ is the molecular weight of water, $R_{0}$ is the gas constant, $T$ is the temperature, $p$ and $p_{w}$ are the total pressure and partial pressure of water, respectively, and $\frac{d p_{w}}{d z}$ is the change of partial pressure of water with distance $z$ from the surface of the drying suspension.

Using diffusion and evaporation equations, mass conservation can now be expressed as follows: 


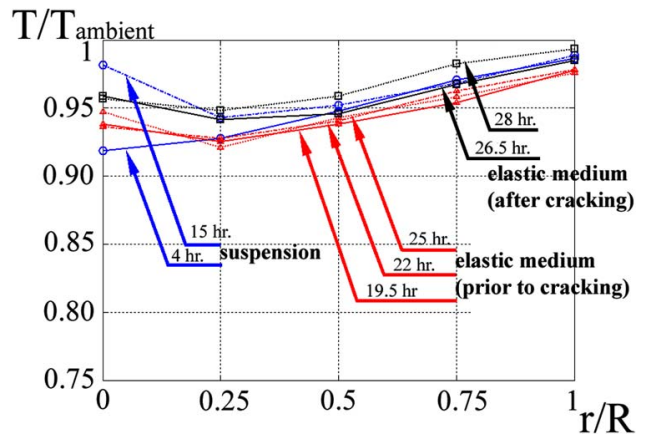

FIG. 4. (Color online) Temperature distribution as a function of time. A steady-state distribution also shown in Fig. 3 is obtained prior to the onset of cracking (19.5-25 h) when the medium attains an elastic state. After cracking, this distribution is disturbed.

$$
\frac{d^{2} C_{w}}{d r^{2}}+\frac{1}{r} \frac{d C_{w}}{d r}=-\left(\frac{D_{e} M_{w}}{t D_{w} R_{0}}\right)\left(\frac{p}{p-p_{w}}\right) \frac{d p_{w}}{d z} \frac{1}{T},
$$

where $t$ is the thickness of the suspension.

In the solution of Eq. (3), experimental data of Fig. 3 will be used. From the figure it is obvious that for all practical purposes the temperature distribution can be taken as constant at steady state with the coffee:water mixture having transformed from a colloidal suspension to a solid with the exception of the periphery, $r=R$, where the mixture remains as a suspension throughout the experiment. The boundary condition at the periphery can be expressed as $C_{w}(R)=C_{w}^{\text {outer }}$. The effect of focusing light at the center can also be seen in the same figure, where a monotonous decrease of temperature towards the center is observed due to evaporative cooling. Furthermore, the change of partial pressure of water with the height from the sample surface, $\frac{d p_{w}}{d z}$, is also taken to be independent of $r$. Hence, the following solution for Eq. (3) is obtained:

$$
C_{w}=-\frac{1}{4}\left(\frac{D_{e} M_{w}}{t D_{w} R_{0}}\right)\left(\frac{p}{p-p_{w}}\right) \frac{d p_{w}}{d z} \frac{1}{T} r^{2}+C_{1} \ln r+C_{2},
$$

where $C_{1}$ and $C_{2}$ are constants. $C_{1}=0$ due to finite $C_{w}$ at the center, and $C_{2}=C_{w}^{\text {outer }}+\frac{1}{4}\left(\frac{D_{e} M_{w}}{t D_{w} R_{0}}\right)\left(\frac{p}{p-p_{w}}\right) \frac{d p_{w}}{d z} \frac{1}{T} R^{2}$. Therefore $C_{w}$ is observed to change with the square of the radial coordinate $r$.

It is to be noted that Eq. (4) corresponds to the steadystate configuration of the coffee:water mixture prior to crack formation. To prove that cracking is indeed delayed until after the equilibrium is attained, a series of temperature measurements are conducted on a drying suspension. Since direct measurement of water concentration within the suspension is not possible, the temperature is measured as the direct indication of evaporation and hence the presence of water and results are presented in Fig. 4. Thermal fluctuations at 4 and $15 \mathrm{~h}$ after casting of the solution are observed to die out when the medium attains an elastic character. Measurements at $19.5,22$, and $25 \mathrm{~h}$ are almost identical, resembling the distribution given in Fig. 3. However, once cracking occurs between 25 and $26.5 \mathrm{~h}$, this steady-state distribution is disturbed, indicating that the predictions of the model can be utilized until the onset of cracking.
Now that the water concentration is known, the stress distribution in the sample can be calculated. Assuming plane stress along the normal to the surface of the suspension and taking the drying material as homogeneous and isotropic, we can adopt a linear elastic approach, where the radial and tangential strains $\epsilon_{r r}$ and $\epsilon_{\theta \theta}$, respectively, are given as

$$
\begin{aligned}
& \epsilon_{r r}-\gamma\left(C_{c r i t}-C_{w}\right)=\frac{1}{E}\left(\sigma_{r r}-\nu \sigma_{\theta \theta}\right), \\
& \epsilon_{\theta \theta}-\gamma\left(C_{c r i t}-C_{w}\right)=\frac{1}{E}\left(\sigma_{\theta \theta}-\nu \sigma_{r r}\right),
\end{aligned}
$$

where $\sigma_{r r}$ and $\sigma_{\theta \theta}$ are radial and tangential stresses, respectively, $\nu$ is Poisson's ratio, and $E$ is the elastic modulus. In driving these equations, it is assumed that normal strains change linearly with the mass concentration of water inside the mixture, $C_{w}$. When this concentration decreases to a critical value $C_{c r i t}$, tensile strains start to build up, and they increase with decreasing water concentration. When the mass concentration of water is higher than $C_{\text {crit }}$, then the medium is a colloidal suspension and, hence, there is no elasticity. The constant of proportionality, $\gamma$, is a positive number that incorporates the effects of interface friction and solute-solvent interactions.

Stress components are then obtained in a way similar to thermal problems of elasticity [30,31], where Eqs. (5) are first solved for stresses, which are then plugged into the following equilibrium equation:

$$
\frac{d \sigma_{r r}}{d r}+\frac{\sigma_{r r}-\sigma_{\theta \theta}}{r}=0
$$

Expressing strains in terms of the radial displacement $u$, the equilibrium equation is rewritten and solved for $u$. Strains and finally stresses are obtained from the displacements

$$
\begin{aligned}
& \sigma_{r r}=\gamma E\left(\frac{1}{R^{2}} \int_{0}^{R}\left(C_{c r i t}-C_{w}\right) \rho d \rho-\frac{1}{r^{2}} \int_{0}^{r}\left(C_{c r i t}-C_{w}\right) \rho d \rho\right) \\
& \sigma_{\theta \theta}= \gamma E\left(-\left(C_{c r i t}-C_{w}\right)+\frac{1}{R^{2}} \int_{0}^{R}\left(C_{c r i t}-C_{w}\right) \rho d \rho\right. \\
&\left.+\frac{1}{r^{2}} \int_{0}^{r}\left(C_{c r i t}-C_{w}\right) \rho d \rho\right)
\end{aligned}
$$

The radial distribution of resulting stresses is shown in Fig. 5, where $\sigma_{r r}$ is always compressive and $\sigma_{\theta \theta}$ is compressive within a circle of radius of approximately $0.6 R$ and tensile outside this circle. Therefore, any cracking should take place outside the compressive region, and moreover, cracks should be oriented along the radial direction, perpendicular to tensile tangential stresses. Experimental observations, also presented in Fig. 5, confirm that there are no cracks in the middle zone of the controlled-drying sample where both principle stresses are compressive. Outside this zone, cracks propagate along the radial direction as predicted by theory. In striking contrast to this, the sample that is left to dry under ambient conditions is covered uniformly with a network of 


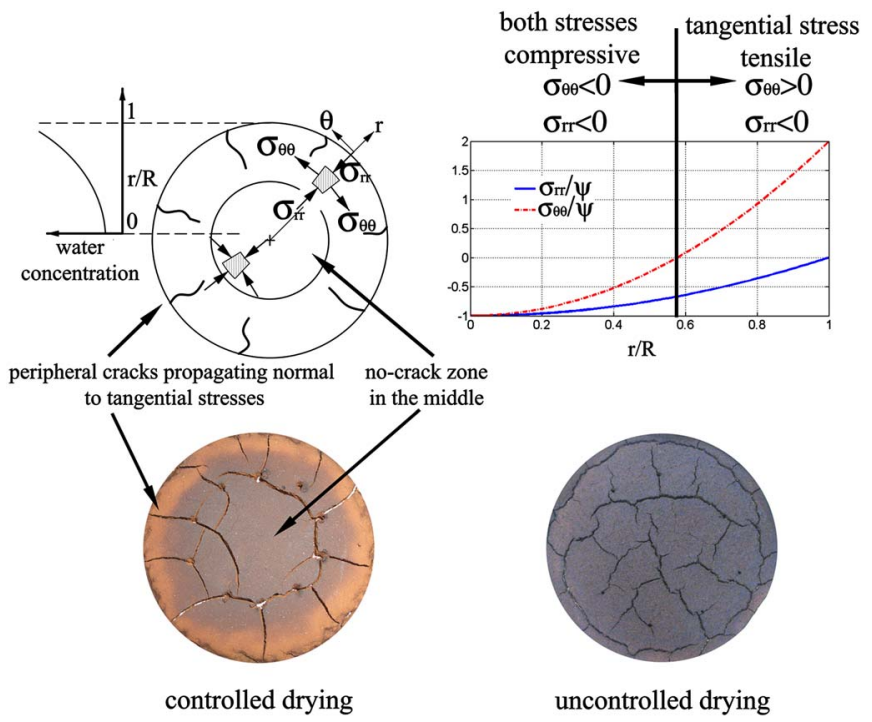

FIG. 5. (Color online) Comparison of crack patterns in controlled and uncontrolled experiments. In the controlled experiment, the perimeter of the circular sample is always kept wet, while the middle portion is dried by focusing light at the center. As predicted by elastic model [Eqs. (7)], a no-crack zone is observed in the middle, where both principal stresses are compressive. Outside this zone, radial stresses remain compressive, whereas tangential stresses become tensile. Hence, cracks in the outer zone tend to propagate along the radial direction. In the uncontrolled experiment, however, cracks are distributed uniformly across the sample. Needles are used in both cases to initiate cracking. Normalization parameter in stress plots is given as $\psi=-\frac{\gamma E R^{2}}{16}\left(\frac{D_{e} M_{w}}{t D_{w} R_{0}}\right)\left(\frac{p}{p-p_{w}}\right) \frac{d p_{w}}{d z} \frac{1}{T}$.

cracks. This simple experiment clearly shows that cracks within the drying suspension of choice obey a maximumopening-stress criterion and kinking should be a mere manifestation of this fact.

As mentioned previously, after the first cracks appear and disturb the uniformity of the medium by creating new evaporation paths [28], $C_{w}$ calculated by Eq. (4) will not anymore determine the stress distribution and successive cracking within the inner compressive circle is evident as shown in Fig. 6.

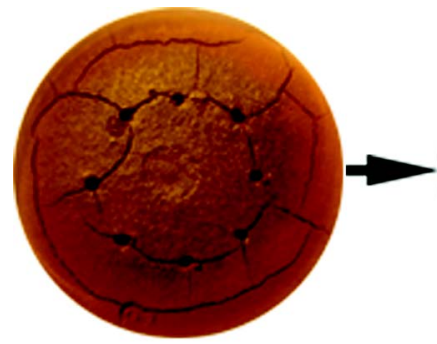

the onset of cracking

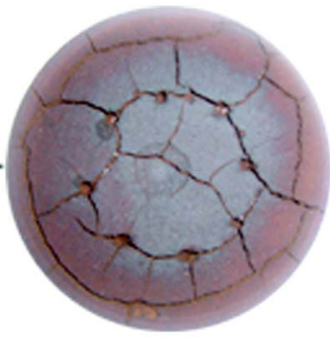

24 hours later
FIG. 6. (Color online) After the first cracks form and disturb the uniformity of the sample, the stress model is not valid anymore. Here $24 \mathrm{~h}$ after the onset of fracture, successive cracking is observed within the no-crack zone.
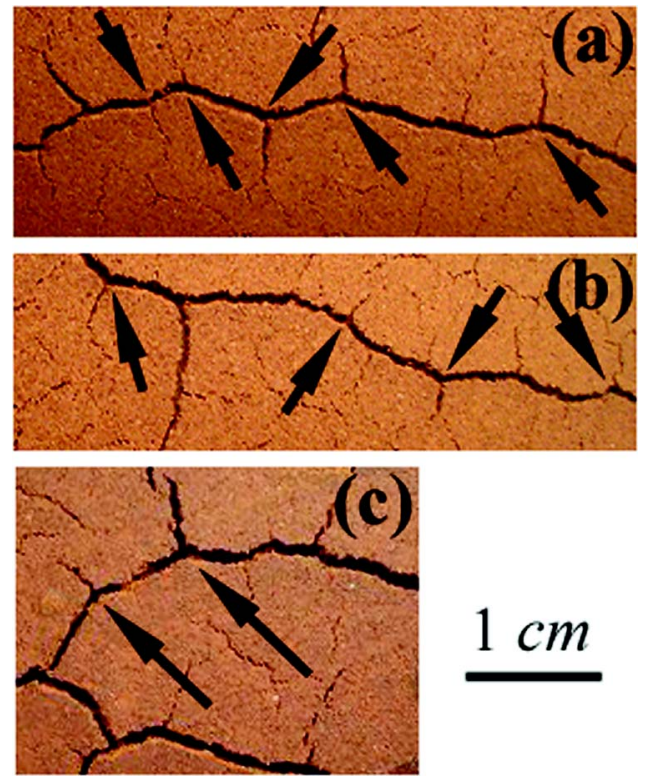

FIG. 7. (Color online) Channeling cracks with sidebranches emanating from the kink points. Kink angles in cracks of (a) and (b) are minor, whereas more severe kinking is exhibited by the crack in (c). If the stress state is nearly equibiaxial, then minor variations in principal stresses ahead of the crack tip lead to moderate kinking, whereas any considerable deviation from equibiaxiality is reflected by a major change of the propagation direction.

\section{SIDE BRANCHING}

Now we will take a closer look at the next step-i.e., sidebranching, as a mechanism for junction formation. Having established the connection between changes in stress state and kinking of cracks, the final question to be answered is the formation of propagation junctions through kink formation. Figure. 7 shows an already cracked sample. In this micrograph one can distinguish between the main channeling cracks that appear thick and tortuous and narrower sidebranches that emanate from the kink points of the main crack. Here, kink locations serve as sharp stress raisers and nucleate the third arm of the propagation junction. To our knowledge, an experimental verification of this simple and straightforward argument is missing in the literature. For this purpose, an in situ observation of the formation of a triple junction is carried out, and the resulting sequence is given in Fig. 8. In this figure, the view is focused on a kink location and the crack is observed under microscope as drying continues. At $0 \mathrm{~h}$, there is only a sharp elbow without sidebranching, and the arrow indicates the nucleation spot of the future sidebranch. After $1 \mathrm{~h}$ the sidebranch already initiates and does not propagate any further for the next $5 \mathrm{~h}$. Between 6 and $7 \mathrm{~h}$ there is an abrupt jump. After $10 \mathrm{~h}$, the crack tip already leaves the field of view, demonstrating clearly the formation of a triple junction during propagation.

\section{CONCLUSION}

In conclusion, we recognize that junctions are crucial for understanding the evolution of desiccation crack networks. 

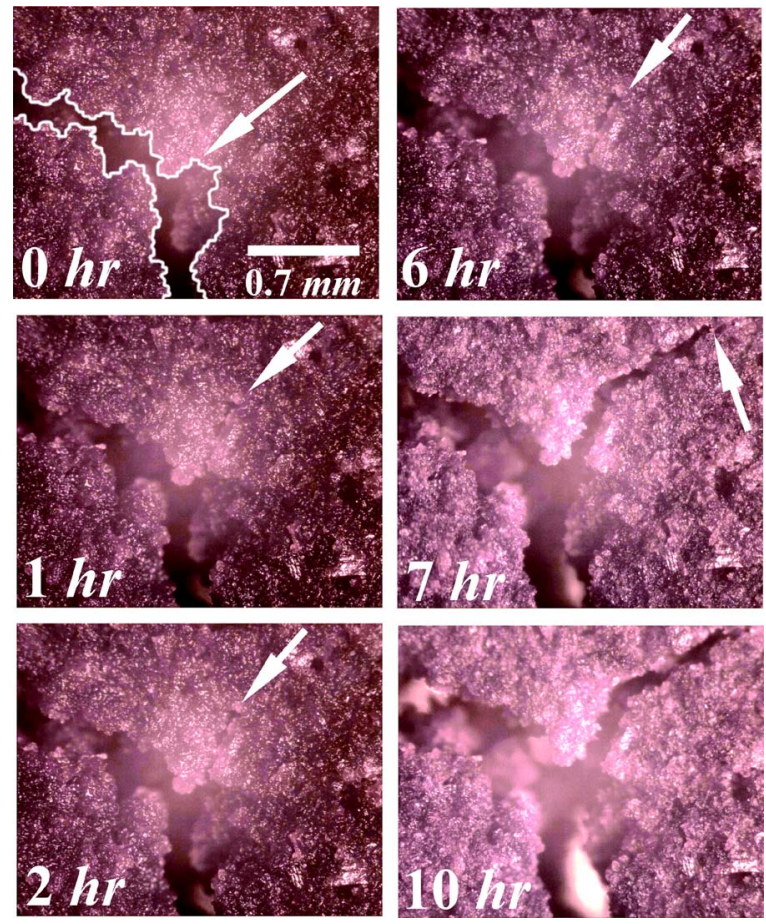

FIG. 8. (Color online) Close-up of the formation of a triple junction at a kink point. The arrow at $0 \mathrm{~h}$ denotes the nucleation spot of the future sidebranch. After $1 \mathrm{~h}$, the sidebranch already initiates and does not propagate any further for the next $5 \mathrm{~h}$. Between 6 and $7 \mathrm{~h}$ there is an abrupt jump. After $10 \mathrm{~h}$, the crack tip already leaves the field of view. At $0 \mathrm{~h}$, crack faces are shown with a white contour to facilitate viewing.

There is a clear distinction between triple junctions formed during initiation phase and those formed during propagation. However, studying the details concerning this distinction has been a difficult task due to the lack of available experimental techniques for separating initiation from propagation. In this work, it has been demonstrated that suppression of propagation can be accomplished by patterning substrate surface with stress raisers. Junctions obtained with and without patterning exhibit a considerable difference. While $120^{\circ}$ junctions during initiation are related to releasing energy stored within the drying medium at minimum cost, angles obtained during propagation are due to joining and sidebranching.

Joining is evident in $90^{\circ}$ junctions. The explanation is straightforward: Due to the absence of tractions on crack surfaces, the ratio of stresses parallel to the crack line to stresses perpendicular to the crack line is maximum in the immediate vicinity of a crack, and hence, when a propagating crack approaches an already existing crack, it diverts from its original path to maximize its opening stress and meets the existing crack perpendicularly.

In contrast to initiation and joining, it is observed that sidebranching is not accompanied by a fixed junction angle. The reason lies in the mechanism of junction formation: A propagating crack does not necessarily divert from its original path only when it enters the immediate vicinity of an existing crack, but there are other irregularities within the drying suspension that lead to an inhomogeneity in the stress state, such as variations in local fracture toughness, interface friction, and solvent concentration. By adjusting its path according to the stresses ahead, a crack sometimes takes turns, called kinks, thereby forming sharp corners. These corners then act as stress raisers and initiate the third arm. The kink angle can also be traced back to the degree of biaxiality of stresses.

The argument about the relation between kink angle and degree of biaxiality is proven by a set of experiments where the stress state within the drying suspension is dictated by imposing certain boundary conditions. It is shown that by having an increasing mass concentration of water from the center to the perimeter of a circular sample, stresses within the drying medium can be determined. While radial stresses turn out to be always compressive, tangential stresses are compressive only within a circular region of 0.6 times the radius of the sample and they become tensile towards the periphery. Therefore it would be reasonable to assume that if crack paths are determined by stresses, cracks in this particular example should propagate only in the outer region and perpendicular to tangential stresses. This expectation was verified by experimental observations. Hence, it can now be argued that kinks, diversions from straight paths frequently observed under ambient (uncontrolled) conditions, can be regarded as adjustments of crack paths to inhomogeneous stresses around them.

Finally a microscopic observation is carried out picturing sidebranching as the growth of a third crack from the sharp corner of a kink, establishing the final link between stresses and junction formation during propagation. Hence, it is concluded that both initiation and propagation are deterministic phenomena, where one can even dictate the path of a crack by manipulating the stress state within the drying suspension. Clear evidence is provided showing that sidebranches form at kink points of a channeling crack, and the broad distribution of junction angles during propagation is presented as an indication of the inhomogeneity in the system. This is found to be in stark contrast to the extremely narrow distribution of junction angles in shrinkage cracks in solid films, where the degree of inhomogeneity is considerably less.
[1] T. Walmann, A. Malthe-Sorenssen, J. Feder, T. Jossang, P. Meakin, and H. H. Hardy, Phys. Rev. Lett. 77, 5393 (1996).

[2] H. Colina and S. Roux, Eur. Phys. J. E 1, 189 (2000).

[3] N. Lecocq and N. Vandewalle, Eur. Phys. J. E 8, 445 (2002).

[4] A. Gorisman and E. Kaplan, Europhys. Lett. 25, 415 (1994).
[5] G. Müller, J. Geophys. Res. 103, 15239 (1998).

[6] K. A. Shorlin, J. R. de Bruyn, M. Graham, and S. W. Morris, Phys. Rev. E 61, 6950 (2000).

[7] E. R. Dufresne, E. I. Corwin, N. A. Greenblatt, J. Ashmore, D. Y. Wang, A. D. Dinsmore, J. X. Cheng, X. S. Xie, J. W. Hutch- 
inson, and D. A. Weitz, Phys. Rev. Lett. 91, 224501 (2003).

[8] L. Pauchard, M. Adda-Bedia, C. Allain, and Y. Couder, Phys. Rev. E 67, 027103 (2003).

[9] Z. Néda, K.-t. Leung, L. Józsa, and M. Ravasz, Phys. Rev. Lett. 88, 095502 (2002).

[10] J. T. Neal, A. M. Langer, and P. F. Kerr, Geol. Soc. Am. Bull. 79, 69 (1968).

[11] J. R. L. Allen, Philos. Trans. R. Soc. London, Ser. B 315, 127 (1987).

[12] R. Weinberger, J. Struct. Geol. 21, 379 (1999).

[13] H.-J. Vogel, H. Hoffmann, and K. Roth, Geoderma 125, 203 (2005).

[14] S. Kitsunezaki, Phys. Rev. E 60, 6449 (1999).

[15] E. A. Jagla, Phys. Rev. E 69, 056212 (2004).

[16] H. Colina, L. de Arcangelis, and S. Roux, Phys. Rev. B 48, 3666 (1993).

[17] U. A. Handge, I. M. Sokolov, and A. Blumen, Phys. Rev. E 61, 3216 (2000).

[18] E. A. Jagla, Phys. Rev. E 65, 046147 (2002).

[19] Y. Chan and Y. Mi, Polymer 45, 3473 (2004).
[20] T. Hornig, I. m. Sokolov, and A. Blumen, Phys. Rev. E 54, 4293 (1996).

[21] K. t. Leung and Z. Néda, Phys. Rev. Lett. 85, 662 (2000).

[22] Z. C. Xia and J. W. Hutchinson, J. Mech. Phys. Solids 48, 1107 (2000).

[23] C. T. Baldwin, J. Sediment. Petrol. 44, 695 (1974).

[24] C. Isenberg, The Science of Soap Films and Soap Bubbles (Dover, New York 1992).

[25] H. Hukusima, Sci. Pap. Inst. Phys. Chem. Res. (Jpn.) 27, 235 (1935).

[26] S. Bohn, L. Pauchard, and Y. Couder, Phys. Rev. E 71, 046214 (2005).

[27] S. Bohn, J. Platkiewicz, B. Andreotti, M. Adda-Bedia, and Y. Couder, Phys. Rev. E 71, 046215 (2005).

[28] C. Allain and L. Limat, Phys. Rev. Lett. 74, 2981 (1995).

[29] K. C. Rolle, Heat and Mass Transfer (Prentice-Hall, Englewood, Cliffs, NJ, 2000).

[30] S. P. Timoshenko and J. N. Goodier, Theory of Elasticity (McGraw-Hill, Singapore 1970).

[31] D. R. Jenkins, Phys. Rev. E 71, 056117 (2005). 